
$\$$ Research Square
Preprints are preliminary reports that have not undergone peer review.
They should not be considered conclusive, used to inform clinical practice, or referenced by the media as validated information.

\title{
Evaluation of Candidate Reference Genes Stability for Gene Expression Analysis by Reverse Transcription QPCR in Clostridium Perfringens
}

\author{
Michele Williams \\ University of Maryland-College Park \\ Mostafa Ghanem ( $\nabla$ mghanem@umd.edu ) \\ University of Maryland-College Park
}

\section{Research Article}

Keywords: genes, reverse transcription quantitative polymerase chain reaction (RT-qPCR), Clostridium perfringens, rRNA gene

Posted Date: December 6th, 2021

DOI: https://doi.org/10.21203/rs.3.rs-1108435/v1

License: () (1) This work is licensed under a Creative Commons Attribution 4.0 International License. Read Full License 


\section{Abstract}

Identification of stable reference genes for normalization purposes is necessary for obtaining reliable and accurate results of reverse transcription quantitative polymerase chain reaction (RT-qPCR) analyses. To our knowledge, no reference gene(s) have been validated for this purpose in Clostridium perfringens. In this study, the expression profile of ten candidate reference genes from three strains of $C$. perfringens were assessed for stability under various experimental conditions using geNorm in qbase+. These stability rankings were then compared to stability assessments evaluated by BestKeeper, NormFinder, delta Ct, and RefFinder algorithms. When comparing all the analyses; gyrA, ftsZ, and recA were identified within the most stable genes under the different experimental conditions and were further tested as a set of reference genes for normalization of alpha toxin gene expression over a 22-hour period. Depending on the condition, $r p \circ A$ and $r$ ho might also be suitable to include as part of the reference set. Although commonly used for the purpose of normalizing RT-qPCR data, the 16S rRNA gene (rrs) was found to be an unsuitable gene to be used as a reference. This work provides a framework for the selection of a suitable stable reference gene set for data normalization of $C$. perfringens gene expression.

\section{Background}

Clostridium perfringens is a ubiquitous microorganism responsible for a wide array of diseases in animals and humans depending on its diverse toxin profile. It has the ability to produce highly resistant endospores that aid in transmitting disease as a result of their persistence in many different environments ${ }^{1}$. In addition to the production of alpha toxin which is present in all $C$. perfringens types, strains associated with various disease syndromes elaborate different toxins ${ }^{2,3}$. Based on these toxin profiles, $C$. perfringens is currently divided into seven toxinotypes- $A$ through $\mathrm{G}^{3}$. The alpha toxin, or phospholipase $\mathrm{C}$, of $C$. perfringens is encoded by the p/c gene and is positively regulated by the two-component VirR/VirS system and the agr cell-cell signaling system ${ }^{4}$. Subtype B isolates cause hemorrhagic enteritis of sheep and produce beta toxin encoded by $c p b$, epsilon toxin encoded by etx, and often perfringolysin $O$ (theta toxin) encoded by the $p f o$ gene $e^{2,5}$. The Agr quorum sensing system regulates expression of $c p b$ and $p f o$, but an as yet unidentified system regulates et $x$ in these strains ${ }^{5}$. Isolates responsible for causing necrotic enteritis of poultry are usually associated with toxinotype $\mathrm{G}$, which elaborate the NetB toxin ${ }^{3,6}$. This toxin is also regulated by the Agr-like quorum sensing system ${ }^{7}$.

One of the barriers to accurate and meaningful gene expression studies is the existence of validated reference genes for normalizing transcripts under specific experimental conditions. Normalization is a key step for producing reliable RT-qPCR assays because it controls for technically and experimentally induced variability in samples allowing for comparisons in target gene expression across these samples ${ }^{8}$. Historically, a single reference gene was chosen for normalization; however, the Minimum Information for Publication of Quantitative Real-Time PCR Experiments (MIQE) guideline indicates that this behavior is no longer acceptable unless proper evidence of constant expression under specific experimental conditions is provided ${ }^{8}$. As pointed out by Hellemans and Vandesomple ${ }^{9}$, performing pilot studies to assess stability of reference genes is an undertaking, and many researchers will rely on literature and colleagues to select reference genes even though the selected gene(s) may not be stable in the experimental system under consideration.

Reference genes for gene expression normalization have been validated for some clostridial species (Clostridioides (Clostridium) difficle $^{10}, C$. Ijungdahlii ${ }^{11}$ and $C$. botulinum ${ }^{12}$ ). Metcalf and colleagues ${ }^{10}$ established that $r r s$, adk, and $r p s J$ ranked as the most stable genes within the set of candidate genes in $C$. difficile when comparing expression during logarithmic growth and stationary growth. For C. botulinum, Kirk et al. established the stability of the $16 \mathrm{~S}$ rRNA geneby determination of the coefficient of variation in Cq values over an 18-hour time course; however, none of the commonly used analytic programs (geNorm ${ }^{13}$, normFinder ${ }^{14}$, BestKeeper $^{15}$, refFinder ${ }^{16}$ ) were utilized to assess stability. For $C$. Ijungdahlii, an important microorganism for biofuel production; gyrA, fotl (formate tetrahydrofolate ligase), and rho were recommended to be used as valid reference genes under the anaerobic growth with five different carbon sources as ranked by geNorm or NormFinder. The transcription level of 16S rRNA gene in this clostridial species, on the other hand, was found to be the least stable when assessed under these tested growth conditions ${ }^{11}$. There have been several studies using RT-qPCR to assess changes in relative gene expression in $C$. perfringens that used different reference genes for the normalization step $^{7,17-20}$. In the Abildgaard study ${ }^{17}$, alpha toxin levels and expression were investigated using TaqMan analysis using gyrA (encoding DNA gyrase) or $r p / L$ (encoding the ribosomal protein L7/L12) as the reference genes for normalization. Both the Saito ${ }^{18}$ and Kawarizadeh ${ }^{20}$ studies utilized the $16 \mathrm{~S}$ rRNA gene as the reference gene. In the Xiao study ${ }^{19}$, the genes $d n a E$ and $s i g H$ were used to normalize expression values, whereas in the $\mathrm{Yu}^{7}$ study, rpoA was used for normalization. As the stability of the reference genes used in these studies was not documented, or single reference genes were used for normalization, it is necessary to establish reference gene 
stability prior to undertaking RT-qPCR analyses for relative gene expression quantification. In our current study, the expression profile and stability of 10 candidate reference genes were assessed in three different strains of $C$. perfringens subjected to three different experimental conditions (length of incubation, oxygen shock, and heat shock) in order to select a set of reference genes that could be used under a variety of experimental conditions. The expression of the plc gene, a virulence gene common to all strains of $C$. perfringens, at various time points during growth was then used to assess the reliability of our selected reference genes for use in normalization. To our knowledge, this is the first study in $C$. perfringens aimed to identify and validate reference genes for normalization of transcript levels for RT-qPCR analysis.

\section{Results}

\section{Selection of the candidate reference genes and their amplification efficiencies.}

Ten genes were identified as potential reference genes from Clostridium species based on their previous use in this or other bacterial species. DNA sequences for eight candidatereference genes previously evaluated for $C$. difficile (adk, gyrA, rho, rpsJ, tpiA, gluD ( $g d h A$ in C. perfringens), rpoA, rrs) ${ }^{10}$ were acquired from NCBI for four Clostridium perfringens strains (ATCC 13124, strain 13, EHE-NE18, and SM101) and aligned in MegAlignPro in order to identify highly (100\%) conserved areas suitable for primer design. Two additional genes ( $f t s Z$ and $r e c A$ ) were chosen as they have been previously validated as suitable reference genes for other bacteria ${ }^{21}$. Species-specific primers for these targets were designed using Primer $3^{22,23}$ (Table 1). Primers sets for $r p o A$ and $r$ rs were used as previously described as they had not been previously validated ${ }^{7,24,25}$.

Primer melting temperatures (Tm) are listed in Table 2 and corresponded to predicted Tms. Amplification specificity was confirmed for all primer pairs by visualizing single PCR amplicons of the correct size on agarose gel electrophoresis (Supplementary Fig. S1). Additionally, single peaks were observed for all 10 primer sets from dissociation curve analysis (Supplementary Fig. S2) indicating to absence of primer-dimers and non-specific amplification products.

Amplicons for each of the ten candidate reference genes were cloned into a cloning vector in order to generate standard curves for the calculation of amplification efficiencies. Amplification efficiencies for these genes ranged from 1.72 (gdhA) to 1.86 (rpsJ) with regression coefficients $\left(R^{2}\right)$ of 0.99 (Table 2 ).

\section{Establishment of experimental conditions.}

We chose to evaluate three varied growth conditions to simulate potential environments the bacterial cells could encounter during pathogenesis. The first condition (referred to as 'incubation period') assessed expression of the candidate reference genes after 4 hours and 9 hours of growth. These time points were chosen to approximate log phase and stationary phase growth; however, due to logistical constraints, real-time bacterial densities could not be monitored during the trial. For this trial, the density of bacterial growth equaled an $\mathrm{OD}_{600}$ of $0.15,0.3$, and 0.78 respectively for $\mathrm{CP}-1, \mathrm{CP}-2$, and $\mathrm{CP}-4$ for the 4 -hour time point and an $\mathrm{OD}_{600}$ of $1.2,1.3$, and 1.4 respectively for $\mathrm{CP}-1, \mathrm{CP}-2$, and $\mathrm{CP}-4$ for the 9-hour time point.

In the second trial, two additional conditions were assessed. One condition (referred to as 'oxygen shock') evaluated expression of the candidate reference genes after a 30-minute exposure to an aerobic environment following 4 hours of growth under normal anaerobic conditions. No measurements of oxygen status were recorded. The other condition (referred to as 'heat shock') evaluated expression of the candidate reference genes after a 30 -minute exposure to $50^{\circ} \mathrm{C}$ following 4 hours of growth under normal $37^{\circ} \mathrm{C}$ growth.

\section{Expression stability of candidate reference genes}

The stability of transcription levels of the candidate reference genes under different stress conditions was initially evaluated by geNorm ${ }^{13}$ within the qbase+ software package, version 3.2 (Biogazelle, Zwijnaarde, Belgium - www.qbaseplus.com). These results were then compared to analyses by additional algorithms- BestKeeper ${ }^{15}$, comparative delta $\mathrm{Ct}^{26}$, normFinder ${ }^{14}$, and RefFinder ${ }^{16}$.

A geNorm pilot experiment utilizing all 10 candidate reference genes produced M-values ranging from 0.69 (gyrA) to 2.0 (gdhA) when assessing stability only for the incubation period experimental condition (Fig. 1). Genes with medium stability, defined as having average geNorm M-values between 0.5 and 1.0, are often seen when heterogeneous sets of samples, such as our bacterial cultures, are evaluated ${ }^{27}$. Four genes fall into the category of medium stability when evaluating the incubation period condition (simulating log and stationary phase) - ftsZ, gyrA, recA, and rpoA or heat shock condition- ftsZ, gyrA, rpoA, and rpsJ (Fig. 1). Under our conditions, oxygen 
shock had the least influence on transcript levels of the candidate reference genes. Six out of the 10 candidate genes were considered stable after being subjected to oxygen shock with $f t s Z, g y r A$. recA, rho, rpsJ, and rpoA having M-values less than 1.0; gyrA, recA, and rho had M-values less than 0.5. Mean $\mathrm{Cq}$ values for an isolate and cycle variation, as indicated by standard deviation of the mean $\mathrm{Cq}$ values, across all conditions for a respective isolate are shown in Table 3. Average Cq values for any given gene would often differ by up to $10 \mathrm{Cq}$ values between isolates for different growth/stress parameters indicating sample heterogeneity. The greatest variation is observed from all genes during comparisons during the incubation period trial (standard deviations range from 0.33 for CP-1 rrs to 6.47 for $\mathrm{CP}-2 \mathrm{rps}$ ). The genes gyrA and recA showed the least variation in cycle number (standard deviation less than 1.0) for each isolate when assessing transcript levels during the oxygen shock and heat shock trials.

To compare the stability ranking determined by qbase+ software to other algorithms, Cq data was submitted to the online tool RefFinder (as found at www.heartcure.com.au/reffinder/). RefFinder ${ }^{16}$ is a comprehensive tool that integrates the output of other computational algorithms by assigning weight to each candidate genes then calculating a final ranking based on the geometric mean of the weighting value for each gene. In this process, the tool provides the output for each of the additional computational programs. Comparisons of the expression stability ranking calculated by RefFinder for geNorm, BestKeeper, NormFinder, and the delta Ct method, as well as the comprehensive RefFinder ranking are shown in Table 4. For most of the algorithms and conditions, the genes $f t s Z$, recA, and gyrA were consistently within the top four ranks. The BestKeeper ${ }^{15}$ computational algorithm showed the most different ranking of the 10 genes under the incubation period condition compared to the other analysis platforms with $r r s$, rho, and $g d h A$ identified as the three most stable genes. Two of these genes ( $r r s$ and $g d h A$ ) were identified among the least stable genes by the other platforms. RefFinder comprehensive rankings identified $f t s Z$, recA, and gyrA consistently within the top four ranks when comparing any of the conditions (incubation period, oxygen shock or heat shock, all conditions together) with geometric means of rank values between 1.41 and 3.71 depending on the growth/stress condition being evaluated. Under two conditions (incubation period and all conditions together), RefFinder identified rho as the third most stable gene with a geometric mean of the rank value at 3.36 and 2.51 , respectively. Under these same conditions, rpoA was not determined to be within the top four most stable genes (rank 9 for incubation period and rank 5 for all conditions together).

\section{Expression profile over time of the alpha toxin gene $(p / c)$}

It has long been recognized that alpha toxin production occurs during active cellular growth ${ }^{17,28-30}$, so evaluating the expression of $p / c$ at various time points during growth should allow us to evaluate the reliability of our selected reference genes for use in normalization of transcript levels. The incubation time points we evaluated were 2.5 hours and 4 hours for simulating early-and mid-log phase, and 6 hours and 22 hours for simulating early- and late-stationary phase. Because of the variability in growth rates between our three $C$. perfringens isolates, it was difficult to achieve the desired growth phase targets (early-log, mid-log, early-stationary) for all three strains when cells were harvested at our experimental time points. The actual $\mathrm{OD}_{600}$ of the cultures used for RNA harvest are shown in Supplementary Table S1. Expression of the plc gene relative to expression of this gene after 22 hours of growth was assessed following normalization with our previously determined set of reference genes ( $f t s Z$, recA, and $g y r A$ ), with these genes individually, with one of the least stable but reliably amplified genes (tpiA), and with rrs as a historically used reference gene (Fig. 2). Our set of reference genes and the same genes individually as reference genes produced similar fold changes in plc expression; however, using the set of reference genes more often produced more consistent results indicated by shorter error bars. When a Student's t-test was used to assess statistical significance $(p \leq 0.05)$ of the changes in expression at 4 hours in relation to the 22-hour time point, a maximal and significant upregulation of p/c transcription was observed when employing $f t s Z$, gyrA, and $r e c A$ either as a set or individually. Although the transcript levels decreased by 6 hours, the level of plc expression was still significantly upregulated when normalizing with the reference set genes or $f t s Z$ and gyrA individually, but not recA individually.

When tpiA, one of the less stably expressed candidate reference genes, was used for normalization; the same pattern of expression was observed but to a greatly depressed level. Expression of $p / c$ at the 4-hour time point was maximal with 9 times less transcript observed when tpiA was used for normalization as compared to the same expression when normalization was performed using the set of three reference genes ( 6-fold increase vs 54 -fold increase, respectively). None of the change in plc expression were found to be significant when tpiA was used in the normalization process.

When $p / c$ expression was normalized with $r r s$, the apparent relative expression of $p / c$ decreased over time instead of increasing during active growth. As such, normalization with the 16S RNA gene produced a different expression profile for p/c than was observed when using our reference set genes (either as a set or individually). Significant upregulation of $p / c$ at the 2.5-hour time point was only noted when rrs was utilized for normalization. 


\section{Discussion}

Clostridium perfringens causes a wide variety of diseases in humans and animals as a result of the toxins it produces ${ }^{2}$. These toxins are regulated by a complex network of signaling pathways and understanding how different environmental conditions modulate the expression of these toxins will provide us with a better understanding of the role of these toxins the development of disease. Reverse transcription qPCR is the gold standard method in studying expression of specific genes and virulence regulation under different environmental conditions ${ }^{31}$, and proper normalization is essential to achieve reliable results ${ }^{8}$. Under the most appropriate normalization strategy, selected reference genes should be verified to be stably expressed under the physiological state or experimental conditions under investigation ${ }^{8}$. To identify suitable genes for normalization of gene expression in $C$. perfringens, we performed the necessary validation analyses under three different conditions that could be relevant during pathogenesis.

In this study, we selected and evaluated ten candidate reference genes (adk, ftsZ, gyrA, gdhA, recA, rho, rpoA, rpsJ, rrs, and tpiA) for use in normalization of transcript levels in $C$. perfringens. As suggested by others, we selected genes representing different functional classes to minimize the likelihood of co-regulation ${ }^{10,32}$. Of these genes; rrs, gyrA, recA, rpoA, rho, ftsZ, and adk have all been experimentally tested for use in other bacteria; however, the stability for each gene was not validated in all studies ${ }^{21}$. For instance, adk has been experimentally tested in 3 different organisms (Bacillus cereus group strains ${ }^{33}, C$. difficile ${ }^{10}$, and $C$. botulinum ${ }^{12}$ ), yet it was only identified as stably expressed in $C$. difficile. Three of the 10 genes investigated ( $g y r A$, $r r s$, and $r p o A$ ) have been used previously as an endogenous control for $C$. perfringens, although validity for these genes was not documented $7,17,18,20,34$.

In our study, we determined the most suitable reference genes for normalization in $C$. perfringens to be ftsZ, gyrA, and recA. This determination was made by assessing the rankings of genes by all the available computational algorithms under the different growth/stress conditions and choosing the genes that ranked highest (within the top four) for the different conditions by each of the computational algorithms. The three genes of our proposed reference set ranked within the top spots under all conditions (analyzed separately and together) by geNorm, NormFinder, and RefFinder (Table 4). These three genes also ranked highest using the delta Ct analysis method when assessing stability under the incubation period trial and when results of all the experimental conditions were combined; whereas gyrA and recA ranked second and third, but $f t s Z$ ranked fifth when assessing expression in the oxygen shock and heat shock experiment. The delta $\mathrm{Ct}$ and geNorm methods are similar in that a stepwise comparison of expression values is made to exclude or rank less favorably genes that fluctuate expression under different conditions. Other genes, especially rho and rpoA, occasionally were ranked in the top 4 , but this ranking was not consistent between conditions and computational algorithms.

The 16S rRNA gene ( $r r s)$ is frequently used as a reference gene for normalization; yet it was not found to be stably expressed in this study. One reason for this instability is likely linked to the considerable variation in rrs transcript levels where they were often extremely abundant $(\mathrm{Cq}$ values $<10)$ and difficult to reproduce consistently as the high abundance makes it difficult to accurately set the baseline $^{13}$. Another reason the rrs gene is not appropriate as a reference gene is that rrs transcript levels were quite abundant in relation to the transcript levels of the other genes including the plc gene as our gene of interest (Cq less than 10 vs. Cq values around 20), and its use is not appropriate as its abundance is overrepresented in relation to the total amount of mRNA in the samples ${ }^{8}$. In fact, when we used rrs as the internal control for normalization of $p / c$, a decreasing upregulation of the alpha toxin gene over time was observed, which is not the same expression profile observed when using the genes in our proposed reference set (Fig. 2).

One hurdle of this study, but one that is likely common for researchers studying bacterial populations with a high degree of heterogeneity, is the inherent variability in gene expression between different strains of the same bacterial species. In C. difficile, a discrepancy between reference gene stability attributable to the heterogeneity of $C$. difficile ribotypes was observed ${ }^{10}$. In this study, we used three $C$. perfringens isolates representing three different toxinotypes- types $\mathrm{B}, \mathrm{E}$, and $\mathrm{G}$. Each isolate showed different growth kinetics with CP-2 (type E) growing most rapidly and CP-1 (type B) growing slowest. Similarly, each isolate differed in the expression levels at the different sampling points. To illustrate this variability in Cq values, we can look at recA and tpiA expression across all the conditions between the different strains. In the oxidative stress experiment, for recA transcripts, there was approximately a $3 \mathrm{Cq}$ value spread between the three strains, but close values between the conditions within a strain as evidenced by the low standard deviations (average $\mathrm{Cq}+/$ - SD values for CP-1, CP-2 and CP-4 are $20.26+/-0.45,23.83+/-0.58$, and $21.92+/-0.30$, respectively; Table 3). On the other hand, for tpiA in the same experiment, there was approximately a $6 \mathrm{Cq}$ value spread between the three strains, but close Cq values between the conditions within a strain as evidenced by the low standard deviations (average $\mathrm{Cq}+/$ - SD values for CP-1, CP-2 and CP-4 are $19.18+/-0.32,26.08+/-0.26$, and $22.91+/-0.67$, respectively; Table 3 ). 
For robust qPCR assays, the general rule-of-thumb is to have near $100 \%$ amplification efficiency as this indicates that the amount of the target doubles at each cycle ${ }^{8}$. The range for a good PCR is $90-110 \%$ with a range of $20 \%(E=1.9$ to 2.1$)$. A potential pitfall of this study is our amplification efficiencies are not within this range; however, they are close to each other. Our PCR efficiencies ranged from $72 \%$ to $86 \%$ with the efficiencies of our chosen reference set between $73 \%$ ( $g y r A)$ to $83.2 \%$ (ftsZ). This range is narrower than the range of efficiency for a "good" qPCR (10 vs $20 \%$ ). So although these efficiencies are lower than we would like, they are similar enough to result in accurate relative quantification ${ }^{35}$.

To test the ability of the proposed set of $C$. perfringens reference genes to be used for normalization, we applied $f t s Z$, gyrA, and recA transcript levels for normalization of the alpha toxin gene $(p / c)$ at different stages of growth. Ohtani and colleagues ${ }^{4}$ noted that toxin gene expression in $C$. perfringens is unique as it is initiated much earlier during growth thereby reaching maximum expression during the exponential phase of growth rather than stationary phase of growth. Ohtani and colleagues ${ }^{4}$, by using Northern blot analysis, attributed this expression pattern to the agrBD system in $C$. perfringens rather than a traditional LuxS-based quorum-sensing mechanism. Using RT-qPCR, Abildgaard and colleagues ${ }^{17}$ observed a similar induction of p/c during early exponential phase with a subsequent decrease in expression occurring by stationary phase. This is the same expression profile observed in this study when using our proposed reference set for normalization. We found that using our proposed reference genes, either in combination or individually, produced similar expression trends over the tested time points from early exponential phase (2.5-hour) to late stationary phase (22-hour). The observance of the transcript level peaking supports previous findings that mRNA expression is induced rather than constitutive $e^{17,29}$.

Selection of stable reference genes for data normalization is required for reliable and accurate RT-qPCR analysis. In this study, we assessed potential reference gene stability of three different $C$. perfringens strains representing three different toxinotypes under three different experimental conditions that could have physiologic relevance. Overall, we recommend the use of $g y r A$, $f t s Z$, and recA as a set of reference genes for use in $C$. perfringens for normalization of gene transcripts in relative expression studies. While we know that ideal universal endogenous control genes have not been identified and likely do not exist ${ }^{13}$ especially for species like $C$. perfringens that are heterogeneous nature, the proposed set of reference genes identified in this study will provide researchers with a good starting point for developing an accurate normalization strategy for their $C$. perfringens-based expression assay. Ideally, these reference genes should be confirmed as stable under other experimental conditions or with additional strains prior to their use.

\section{Methods}

\section{Strains, media, and growth conditions:}

Two strains of $C$. perfringens were purchased from ATCC belonging to toxintype B (ATCC 3626, CP-1) and toxintype E (ATCC 27324, CP2). A third strain containing netB (C103-100, CP-4) was obtained from Auburn University (Ken Macklin, extension specialist and professor) that originated from the Mitchem-Sparks Regional Diagnostic Laboratory in Boaz, Alabama. This netB-containing isolate is classified as subtype $\mathrm{G}$ in the recently expanded toxin-based typing scheme ${ }^{3}$.

Bacterial cultures were grown on perfringens agar (HiMedia, Kennett Square, PA) for routine maintenance and in BHI (Criterion, Hardy Diagnostics, Santa Maria, CA) broth during experimental assays. An anaerobic environment was maintained in standard sized growth chambers containing gas-generating sachets with anaerobic indicators (Becton, Dickinson and Company; Franklin Lakes, NJ). Starter $\mathrm{BHI}$ cultures were inoculated from plates and allowed to grow anaerobically overnight ( 16 hours) at $37^{\circ} \mathrm{C}$. Trials were carried out as two experiments. In Experiment 1 for the incubation period trial, $10 \mathrm{ml} \mathrm{BHl}$ cultures were inoculated using $200 \mu \mathrm{l}$ of the appropriate starter culture, then allowed to grow 4 hours to approximate the logarithmic phase (target $\mathrm{OD}_{600} 0.3-0.4$ ) or 9 hours to approximate the stationary phase (target $\mathrm{OD}_{600} 1.2-1.4$ ). After the appropriate time points, the cultures were divided into 2 ml aliquots, centrifuged at $3,260 \mathrm{Xg}$ for 4 minutes, then pellets were stored at $-80^{\circ} \mathrm{C}$. For experiment 2, the oxygen shock and heat shock trials, all cultures were inoculated and incubated as for the incubation period samples in Experiment 1. At 4-hours, the control samples were divided into $1.5 \mathrm{ml}$ aliquots, centrifuged at $14,000 \mathrm{xg}$ for 2 minutes, then stored at $-80^{\circ} \mathrm{C}$ or immediately processed for RNA extraction. The oxygen shock samples were removed from the anaerobic growth chamber and incubated aerobically at $37^{\circ} \mathrm{C}$ for 25 minutes, after which time $1.5 \mathrm{ml}$ aliquots were centrifuged at $14,000 \mathrm{xg}$ for 2 minutes, then stored at $-80^{\circ} \mathrm{C}$ or immediately processed for RNA extraction. For the heat shock samples, cultures were transferred to a $50^{\circ} \mathrm{C}$ water bath for 30 minutes $^{36}$, after which time $1.5 \mathrm{ml}$ aliquots were centrifuged at $14,000 \times \mathrm{g}$ for 2 minutes, then stored at $-80^{\circ} \mathrm{C}$ or immediately processed for RNA extraction. 


\section{Primers:}

Ten genes were identified as potential reference genes from the pertinent Clostridium sp. literature (Table 1). Eight $C$. perfringensspecific primers were designed for the genes utilized by Metcalf et $a f^{10}$ for $C$. difficile. Primers were designed using Primer $3 w e b^{22,23}$ with the following parameters: optimal primer size- $20 \mathrm{bp}$ (range $18 \mathrm{bp}$ to $23 \mathrm{bp}$ ); $\mathrm{Tm}-59^{\circ} \mathrm{C}$ (range $57^{\circ} \mathrm{C}$ to $62^{\circ} \mathrm{C}$ ), GC content- $50 \%$ (range $30 \%$ to $70 \%$ ). Using Primer-BLAST against the non-redundant (nr) nucleotide database, potential primers were assessed to check that $C$. perfringens entries were the only match at $>95 \%$ similarity for the primer set. Primers sets for $r p o A$ and $r r s$ were used as previously described ${ }^{7,24,25}$. All primers were synthesized by Integrated DNA Technologies (IDT, Coralville, IA) and purified by standard desalting methods.

\section{Determination of PCR efficiency}

All 10 putative reference genes were amplified, purified (GeneJet PCR Purification Kit, Life Technologies, Carlsbad, CA), and cloned into pMiniT 2.0 cloning vector (NEB PCR Cloning Kit, New England Biolabs, Ipswitch, MA) to establish a standard curve for the determination of the PCR efficiency. Clones were transformed into E. coli (NEB 10-beta competent $E$. coli, New England Biolabs), and colonies were screened by PCR. Bacterial cells containing plasmids with proper inserts were expanded in LB amended with $100 \mathrm{mg} / \mathrm{ml}$ carbenicillin to facilitate extraction of the plasmids (Monarch Plasmid Miniprep kit, New England Biolabs). Inserts were verified by sequencing. Plasmid DNA concentrations were measured spectrophotometrically using a Nanodrop 1000 (Thermo Scientific, Wilmington, DE). A 6-point 10 -fold dilution series (concentration ranges from $10 \mathrm{ng} / \mu \mathrm{l}$ to $100 \mathrm{fg} / \mu \mathrm{l}$ ) was prepared. Ten $\mu \mathrm{l}$ reactions were prepared in quadruplicate for each reference gene-containing plasmid using PowerUp SYBR Green Master Mix (Applied Biosystems) and $500 \mathrm{nM}$ of each primer in the respective primer set. Amplification was performed using a CFX-96 Connect real time thermal cycler (Bio-Rad, Hercules, CA) with the following cycling parameters: $50^{\circ} \mathrm{C}$ UDG activation for 2 minutes, $95^{\circ} \mathrm{C}$ DNA polymerase unlock step for 2 minutes, followed by 40 cycles of $95^{\circ} \mathrm{C}$ for 15 seconds, $61^{\circ} \mathrm{C}$ for 15 seconds, $72{ }^{\circ} \mathrm{C}$ for 1 minute with fluorescent images captured at the completion of each cycle. The amplification was immediately followed by a dissociation curve analysis to check for primer-dimer formation and any nonspecific amplification products.

\section{RNA isolation and reverse transcription}

Total RNA was isolated from the stored pellets of $C$. perfringens isolates harvested at logarithmic and stationary phases of growth using the Promega SV Total RNA kit (Promega Corporation, Madison, WI) following the manufacturer's protocol for Isolation of RNA from Gram-Positive and Gram-Negative Bacteria with a few modifications. Pellets were resuspended in $100 \mu$ of freshly prepared TE containing $20 \mathrm{mg} / \mathrm{ml}$ lysozyme and incubated at room temperature for 10 minutes. Purified RNA was eluted with $100 \mu \mathrm{l}$ nuclease-free water. A post-elution DNase treatment was performed (TURBO DNA-free kit, Invitrogen). RNA concentrations were assessed spectrophotometrically using a Nanodrop 1000 (Thermo Scientific, Wilmington, DE) prior to normalization of RNA concentration. Concentration of normalized RNA was subsequently verified by Nanodrop to be within $10 \%$ of each other.

\section{Reference gene validation}

Ten $\mu \mathrm{l}$ reactions were prepared in duplicate for each $C$. perfringens cDNA with each of 10 potential reference genes using PowerUp SYBR Green Master Mix (Applied Biosystems) and $500 \mathrm{nM}$ of each primer of the respective primer set. No reverse transcriptase controls were included for each RNA isolation. Amplification was performed in clear low profile plates using a CFX-96 Connect real time thermal cycler (Bio-Rad, Hercules, CA) with the following cycling parameters: $50^{\circ} \mathrm{C}$ UDG activation for 2 minutes, $95^{\circ} \mathrm{C}$ DNA polymerase unlock step for 2 minutes, followed by 40 cycles of $95^{\circ} \mathrm{C}$ for $15 \mathrm{sec}, 61^{\circ} \mathrm{C}$ for 15 seconds, $72{ }^{\circ} \mathrm{C}$ for 1 minute with fluorescent images captured at the completion of each cycle. Amplification was immediately followed by dissociation curve analyses to check for primer-dimer formation and any nonspecific amplification products.

Cq values for each candidate reference gene were analysed for stability using geNorm ${ }^{13}$ within the qbase+ software package, version 3.2 (Biogazelle, Zwijnaarde, Belgium - www.qbaseplus.com). Comparisons of stability rankings were made following additional computational analyses using BestKeeper ${ }^{15}$, comparative delta $\mathrm{Ct}^{26}$, normFinder ${ }^{14}$, and RefFinder ${ }^{16}$.

\section{Alpha toxin gene $(p / c)$ qPCR}

The set of reference genes identified by geNorm to be most stable under these stress conditions were used to normalize expression of plc in response to growth phase. Primers were designed using the same parameters as for the candidate reference genes to amplify a 
$174 \mathrm{bp}$ fragment of the plc gene of $C$. perfringens (forward primer 5'- CTG ACA CAG GGG AAT CAC AA- 3', reverse primer 5'- CAT GTC CTG CGC TAT CAA CG- $3^{\prime}$ ). As alpha toxin has been shown to be regulated by cell density mechanisms, the growth time points we assessed were 2.5 hours (early-log), 4 hours (mid-log), 6 hours (early stationary), and 22-hour (late stationary). Bacteria were grown and harvested at each experimental time point as previously described. Procedures for RNA isolation, cDNA synthesis, and RT-qPCR conditions were the same as for the reference gene validation experiments. Stability of the reference genes under these growth conditions were confirmed with geNorm (qbase+). Normalized gene expression of $p / c$ was determined using the multi-gene normalization quantification model ${ }^{27}$ and statistical significance of $p / c$ expression due to growth phase in relation to the 22-hour time point simulating stationary phase when alpha toxin gene expression should be minimal was assessed using a two-tailed Student's ttest $(p \leq 0.05)$.

\section{Declaration}

\section{Acknowledgements}

This study was funded by Virginia-Maryland Regional College of Veterinary Medicine seed grant for the year 2020. We would like to thank Ken Macklin for graciously contributing the netB-positive isolate, Sean Riley for technical assistance with RT-qPCR assay design, and Margie Lee for critical review of the manuscript.

\section{Author contributions}

Both M.G. and M.L.W. conceived and designed the experiments. M.L.W. executed the experiments and the analysis. M.G advised on the data analysis. M.G. acquired the funding. M.L.W. and M.G. wrote the manuscript and coordinated its revision. Both authors approved the final version of the manuscript.

\section{Additional Information}

Supplementary information: Supplementary data file included.

Competing interests: The authors declare no competing interests.

\section{References}

1. Shen, A., Edwards, A. N., Sarker, M. R. \& Paredes-Sabja, D. Sporulation and germination in clostridial pathogens. Microbiol. Spectr. 7, 10.1128/microbiolspec.GPP3-0017-2018 (2019).

2. Uzal, F. A. et al. Towards an understanding of the role of Clostridium perfringens toxins in human and animal disease. Future Microbiol. 9, 361-377 (2014).

3. Rood, J. I. et al. Expansion of the Clostridium perfringens toxin-based typing scheme. Anaerobe 53, 5-10 (2018).

4. Ohtani, K. et al. Virulence gene regulation by the agr system in Clostridium perfringens. J. Bacteriol. 191, 3919-3927 (2009).

5. Chen, J. \& McClane, B. A. Role of the Agr-like quorum-sensing system in regulating toxin production by Clostridium perfringens type B strains CN1793 and CN1795. Infect. Immun. 80, 3008-3017 (2012).

6. Keyburn, A. L. et al. NetB, a new toxin that is associated with avian necrotic enteritis caused by Clostridium perfringens. PLOS Pathog. 4, e26 (2008).

7. Yu, Q. et al. The Agr-like quorum sensing system is required for pathogenesis of necrotic enteritis caused by Clostridium perfringens in poultry. Infect. Immun. 85, e00975-16 (2017).

8. Bustin, S. A. et al. The MIQE guidelines: Minimum information for publication of quantitative real-time PCR experiments. Clin. Chem. 55, 611-622 (2009).

9. Hellemans, J. \& Vandesompele, J. Selection of reliable reference genes for RT-qPCR analysis. Methods Mol. Biol. Clifton NJ 1160, 19-26 (2014).

10. Metcalf, D., Sharif, S. \& Weese, J. S. Evaluation of candidate reference genes in Clostridium difficile for gene expression normalization. Anaerobe 16, 439-443 (2010).

11. Liu, J., Tan, Y., Yang, X., Chen, X. \& Li, F. Evaluation of Clostridium ljungdahlii DSM 13528 reference genes in gene expression studies by qRT-PCR. J. Biosci. Bioeng. 116, 460-464 (2013). 
12. Kirk, D. G., Palonen, E., Korkeala, H. \& Lindström, M. Evaluation of normalization reference genes for RT-qPCR analysis of spo0A and four sporulation sigma factor genes in Clostridium botulinum Group I strain ATCC 3502. Anaerobe 26, 14-19 (2014).

13. Vandesompele, J. et al. Accurate normalization of real-time quantitative RT-PCR data by geometric averaging of multiple internal control genes. Genome Biol. 3, research0034.1 (2002).

14. Andersen, C. L., Jensen, J. L. \& Ørntoft, T. F. Normalization of real-time quantitative reverse transcription-PCR data: A model-based variance estimation approach to identify genes suited for normalization, applied to bladder and colon cancer data sets. Cancer Res. 64, 5245-5250 (2004).

15. Pfaffl, M. W., Tichopad, A., Prgomet, C. \& Neuvians, T. P. Determination of stable housekeeping genes, differentially regulated target genes and sample integrity: BestKeeper - Excel-based tool using pair-wise correlations. Biotechnol. Lett. 26, 509-515 (2004).

16. Xie, F., Xiao, P., Chen, D., Xu, L. \& Zhang, B. miRDeepFinder: a miRNA analysis tool for deep sequencing of plant small RNAs. Plant Mol. Biol. (2012) doi:10.1007/s11103-012-9885-2.

17. Abildgaard, L., Schramm, A., Rudi, K. \& Højberg, O. Dynamics of plc gene transcription and a-toxin production during growth of Clostridium perfringens strains with contrasting a-toxin production. Vet. Microbiol. 139, 202-206 (2009).

18. Saito, R., Talukdar, P. K., Alanazi, S. S. \& Sarker, M. R. RelA/DTD-mediated regulation of spore formation and toxin production by Clostridium perfringens type A strain SM101. Microbiol. Read. Engl. 164, 835-847 (2018).

19. Xiao, Y., van Hijum, S. A. F. T., Abee, T. \& Wells-Bennik, M. H. J. Genome-wide transcriptional profiling of Clostridium perfringens SM101 during sporulation extends the core of putative sporulation genes and genes determining spore properties and germination characteristics. PloS One 10, e0127036 (2015).

20. Kawarizadeh, A., Tabatabaei, M., Hosseinzadeh, S., Farzaneh, M. \& Pourmontaseri, M. The effects of probiotic Bacillus coagulans on the cytotoxicity and expression of alpha toxin gene of Clostridium perfringens type A. Anaerobe 59, 61-67 (2019).

21. Rocha, D. J. P., Santos, C. S. \& Pacheco, L. G. C. Bacterial reference genes for gene expression studies by RT-qPCR: survey and analysis. Antonie Van Leeuwenhoek 108, 685-693 (2015).

22. Untergasser, A. et al. Primer3-new capabilities and interfaces. Nucleic Acids Res. 40, e115 (2012).

23. Koressaar, T. \& Remm, M. Enhancements and modifications of primer design program Primer3. Bioinformatics 23, 1289-1291 (2007).

24. Matsuda, K. et al. Establishment of an analytical system for the human fecal microbiota, based on reverse transcriptionquantitative PCR targeting of multicopy rRNA molecules. Appl. Environ. Microbiol. 75, 1961-1969 (2009).

25. Kikuchi, E., Miyamoto, Y., Narushima, S. \& Itoh, K. Design of species-specific primers to identify 13 species of Clostridium harbored in human intestinal tracts. Microbiol. Immunol. 46, 353-358 (2002).

26. Silver, N., Best, S., Jiang, J. \& Thein, S. L. Selection of housekeeping genes for gene expression studies in human reticulocytes using real-time PCR. BMC Mol. Biol. 7, 33 (2006).

27. Hellemans, J., Mortier, G., De Paepe, A., Speleman, F. \& Vandesompele, J. qBase relative quantification framework and software for management and automated analysis of real-time quantitative PCR data. Genome Biol. 8, R19 (2007).

28. Möllby, R., Holme, T., Nord, C. E., Smyth, C. J. \& Wadström, T. Production of phospholipase C (alpha-toxin), haemolysins and lethal toxins by Clostridium perfringens types A to D. J. Gen. Microbiol. 96, 137-144 (1976).

29. Bullifent, H. L. et al. The level of expression of a-toxin by different strains of Clostridium perfringensis dependent on differences in promoter structure and genetic background. Anaerobe 2, 365-371 (1996).

30. Bullifent, H. L., Moir, A. \& Titball, R. W. The construction of a reporter system and use for the investigation of Clostridium perfringens gene expression. FEMS Microbiol. Lett. 131, 99-105 (1995).

31. Aviv, G. \& Gal-Mor, O. Real-time reverse transcription PCR as a tool to study virulence gene regulation in bacterial pathogens. in Host-Pathogen Interactions: Methods and Protocols (eds. Medina, C. \& López-Baena, F. J.) 23-32 (Springer, 2018). doi:10.1007/978-1-4939-7604-1_3.

32. Gomes, A. É. I. et al. Selection and validation of reference genes for gene expression studies in Klebsiella pneumoniae using reverse transcription quantitative real-time PCR. Sci. Rep. 8, 9001 (2018).

33. Reiter, L., Kolst $\varnothing$, A.-B. \& Piehler, A. P. Reference genes for quantitative, reverse-transcription PCR in Bacillus cereus group strains throughout the bacterial life cycle. J. Microbiol. Methods 86, 210-217 (2011).

34. Hiscox, T. J., Ohtani, K., Shimizu, T., Cheung, J. K. \& Rood, J. I. Identification of a two-component signal transduction system that regulates maltose genes in Clostridium perfringens. Anaerobe 30, 199-204 (2014).

Page 9/15 
35. VanGuilder, H. D., Vrana, K. E. \& Freeman, W. M. Twenty-five years of quantitative PCR for gene expression analysis. BioTechniques 44, 619-626 (2008).

36. Heredia, N. L., Labbé, R. G. \& García-Alvarado, J. S. Alteration in sporulation, enterotoxin production, and protein synthesis by Clostridium perfringens type A following heat shock. J. Food Prot. 61, 1143-1147 (1998).

\section{Tables}

Table 1. Candidate reference genes, functional groups, primer sequences, and expected amplicon sizes. Fragment sizes are based on alignment to C. perfringens ATCC 13124.

\begin{tabular}{|c|c|c|c|c|}
\hline \multirow[t]{2}{*}{ Gene } & Functional & Sequence $\left(5^{\prime} \rightarrow 3^{\prime}\right)$ & Size (bp) & Reference \\
\hline & Category & & & \\
\hline \multirow[t]{2}{*}{$a d k$} & \multirow[t]{2}{*}{ Nucleotide metabolism } & F: TGGAAAATGTGATCTTTGCGGA & \multirow[t]{2}{*}{173} & This study \\
\hline & & R: AGACCTCGTTTATTGCTTGTGT & & \\
\hline \multirow[t]{2}{*}{ gyrA } & \multirow[t]{2}{*}{ DNA replication } & F: GAGGTGGTAAGGGAATACAAGC & \multirow[t]{2}{*}{167} & This study \\
\hline & & R: TGTTCCCTTAGCAGTTCTTCCT & & \\
\hline \multirow[t]{2}{*}{ recA } & \multirow[t]{2}{*}{ DNA replication } & F: CTGGTAAAACAACAGTGGCTTT & \multirow[t]{2}{*}{167} & This study \\
\hline & & R: AGCTTGTTCTCCTGTATCTGGT & & \\
\hline \multirow[t]{2}{*}{ rho } & \multirow[t]{2}{*}{ Transcription } & F: TGAAAGACCAGAAGAAGTAACGG & \multirow[t]{2}{*}{150} & This study \\
\hline & & R: АСАTCTCTTCCTTGTTCAACCA & & \\
\hline \multirow[t]{2}{*}{ rpsJ } & \multirow[t]{2}{*}{ Translation } & F: ACAGGAGCAAAGGTTGTTGG & \multirow[t]{2}{*}{150} & This study \\
\hline & & R: TGGTGATGGATTAACGATGTCT & & \\
\hline \multirow[t]{2}{*}{ tpiA } & \multirow[t]{2}{*}{ Energy metabolism } & F: GCATTCACTGGAGAAATCGCT & \multirow[t]{2}{*}{153} & This study \\
\hline & & R: TGGAGTTAAGTTGTGTGCGA & & \\
\hline \multirow[t]{2}{*}{ fts $Z$} & \multirow[t]{2}{*}{ Cell division } & F: AGGTAATTGGATGTGGAGGC & \multirow[t]{2}{*}{123} & This study \\
\hline & & R: GGGCATGAGATAGTGTTAAGGC & & \\
\hline \multirow[t]{2}{*}{$g d h A$} & \multirow[t]{2}{*}{ Energy metabolism } & F: GGTGGAGTAGCAACATCAGC & \multirow[t]{2}{*}{143} & This study \\
\hline & & R: TGACCATATTCAGAAGCTGCA & & \\
\hline \multirow[t]{2}{*}{ rроA } & \multirow[t]{2}{*}{ Transcription } & F: TTACCTGGAGTGGCTCCAAC & \multirow[t]{2}{*}{176} & 7 \\
\hline & & R: ACACCTGGTCCTTGAGCATC & & \\
\hline \multirow[t]{2}{*}{ rrs } & \multirow[t]{2}{*}{ Translation } & F: GGGGGTTTCAACACCTCC & \multirow[t]{2}{*}{170} & 24 \\
\hline & & R: GCAAGGGATGTCAAGTGT & & 25 \\
\hline
\end{tabular}

Table 2. Features of the candidate reference genes 


\begin{tabular}{|lllll|}
\hline Gene & $\begin{array}{l}\text { Coefficient of Determination } \\
\left(\mathrm{R}^{2}\right)\end{array}$ & $\begin{array}{l}\text { PCR Efficiency [E (\%)]- CFX } \\
\text { Maestro }\end{array}$ & $\begin{array}{l}\text { PCR Efficiency [E (SE)]- } \\
\text { qbase+ }\end{array}$ & $\begin{array}{l}\text { Melting Temperature } \\
\left({ }^{\circ} \mathrm{C}\right)\end{array}$ \\
\hline adk & 0.997 & $1.82(81.5)$ & $1.81(0.012)$ & 76.0 \\
\hline ftsZ & 0.996 & $1.83(83.2)$ & $1.82(0.014)$ & 76.5 \\
\hline gdhA & 0.996 & $1.72(72.4)$ & $1.71(0.010)$ & 74.5 \\
\hline gyrA & 0.997 & $1.73(73.0)$ & $1.73(0.009)$ & 76.5 \\
\hline recA & 0.998 & $1.81(81.0)$ & $1.79(0.013)$ & 78.5 \\
\hline rho & 0.995 & $1.83(82.8)$ & $1.81(0.016)$ & 75.0 \\
\hline rpsJ & 0.997 & $1.86(86.1)$ & $1.83(0.005)$ & 77.5 \\
\hline tpiA & 0.998 & $1.82(82.4)$ & $1.81(0.004)$ & 77.0 \\
\hline rpoA & 0.997 & $1.75(74.8)$ & $1.74(0.013)$ & 76.0 \\
\hline rrs & 0.997 & $1.78(77.7)$ & $1.76(0.014)$ & 84.0 \\
\hline
\end{tabular}

Table 3. Cycle variation across all samples for each growth/stress condition. 


\begin{tabular}{|c|c|c|c|c|c|}
\hline \multirow[t]{2}{*}{ Gene } & \multirow[t]{2}{*}{ Isolate } & \multicolumn{4}{|l|}{ Average $\mathrm{Cq}+/-\mathrm{SD}$} \\
\hline & & Incubation Period & Oxygen Shock & Heat Shock & All Conditions \\
\hline \multirow[t]{3}{*}{$a d k$} & $\mathrm{CP}-1$ & $19.04+/-4.82$ & $18.22+/-0.92$ & $17.50+/-1.08$ & $18.22+/-3.23$ \\
\hline & CP-2 & $20.79+/-4.74$ & $25.42+/-1.62$ & $26.28+/-2.47$ & $24.42+/-3.61$ \\
\hline & CP-4 & $21.26+/-3.10$ & $19.31+/-1.76$ & $19.29+/-1.11$ & $19.81+/-2.43$ \\
\hline \multirow[t]{3}{*}{$f t s Z$} & CP-1 & $18.93+/-1.91$ & $17.80+/-1.04$ & $18.31+/-1.17$ & $18.45+/-1.48$ \\
\hline & CP-2 & $19.32+/-2.22$ & $20.36+/-0.40$ & $21.02+/-1.06$ & $20.28+/-1.75$ \\
\hline & $\mathrm{CP}-4$ & $20.88+/-2.48$ & $20.07+/-0.59$ & $20.46+/-0.97$ & $20.67+/-1.69$ \\
\hline \multirow[t]{3}{*}{$g d h A$} & $\mathrm{CP}-1$ & $23.11+/-2.48$ & $31.63+/-2.25$ & $34.69+/-0$ & $26.76+/-4.84$ \\
\hline & CP-2 & $21.77+/-1.52$ & $30.43+/-1.23$ & $33.81+/-4.27$ & $28.45+/-6.27$ \\
\hline & $\mathrm{CP}-4$ & $24.72+/-3.14$ & $33.40+/-3.81$ & $33.78+/-1.17$ & $29.75+/-5.60$ \\
\hline \multirow[t]{3}{*}{ gyrA } & $\mathrm{CP}-1$ & $17.94+/-2.71$ & $18.09+/-0.74$ & $18.01+/-0.78$ & $17.97+/-1.79$ \\
\hline & CP-2 & $16.98+/-2.72$ & $21.21+/-0.77$ & $21.36+/-0.89$ & $19.72+/-2.89$ \\
\hline & CP-4 & $19.28+/-2.76$ & $19.27+/-0.17$ & $19.54+/-0.38$ & $19.41+/-1.77$ \\
\hline \multirow[t]{3}{*}{ recA } & $\mathrm{CP}-1$ & $18.21+/-1.91$ & $20.26+/-0.45$ & $19.43+/-0.58$ & $19.18+/-1.55$ \\
\hline & CP-2 & $17.63+/-2.07$ & $23.83+/-0.58$ & $23.45+/-0.21$ & $21.31+/-3.30$ \\
\hline & CP-4 & $18.91+/-1.83$ & $21.92+/-0.30$ & $21.34+/-0.55$ & $20.50+/-1.79$ \\
\hline \multirow[t]{3}{*}{ rho } & CP-1 & $19.64+/-0.60$ & $20.58+/-0.67$ & $21.15+/-1.03$ & $20.50+/-1.05$ \\
\hline & CP-2 & $20.20+/-2.13$ & $23.40+/-0.86$ & $23.96+/-1.40$ & $22.51+/-2.48$ \\
\hline & $\mathrm{CP}-4$ & $20.83+/-1.77$ & $22.47+/-0.45$ & $23.57+/-1.62$ & $22.33+/-1.97$ \\
\hline \multirow[t]{3}{*}{ rpsJ } & $\mathrm{CP}-1$ & $16.00+/-4.04$ & $16.29+/-0.62$ & $16.66+/-0.70$ & $16.29+/-2.61$ \\
\hline & CP-2 & $11.62+/-6.47$ & $22.24+/-1.41$ & $21.95+/-1.12$ & $20.05+/-3.93$ \\
\hline & $\mathrm{CP}-4$ & $17.93+/-2.84$ & $18.50+/-0.26$ & $19.36+/-1.09$ & $18.66+/-2.03$ \\
\hline \multirow[t]{3}{*}{ tpiA } & CP-1 & $19.37+/-2.08$ & $19.18+/-0.32$ & $21.04+/-2.12$ & $20.05+/-2.37$ \\
\hline & CP-2 & $21.47+/-3.48$ & $26.08+/-0.26$ & $26.75+/-0.89$ & $24.55+/-3.93$ \\
\hline & $\mathrm{CP}-4$ & $20.96+/-1.64$ & $22.91+/-0.67$ & $22.99+/-0.82$ & $22.29+/-1.60$ \\
\hline \multirow[t]{3}{*}{ rpoA } & CP-1 & $17.24+/-2.82$ & $15.08+/-1.06$ & $15.84+/-0.81$ & $16.12+/-2.14$ \\
\hline & CP-2 & $15.24+/-4.71$ & $19.07+/-1.47$ & $19.32+/-1.72$ & $18.99+/-2.34$ \\
\hline & CP-4 & $19.20+/-3.26$ & $17.01+/-0.36$ & $17.41+/-0.46$ & $18.00+/-2.31$ \\
\hline \multirow[t]{3}{*}{ rrs } & $\mathrm{CP}-1$ & $4.66+/-0.33$ & $5.47+/-3.10$ & $6.70+/-3.00$ & $5.30+/-2.47$ \\
\hline & CP-2 & $6.79+/-1.74$ & $4.15+/-1.27$ & $7.70+/-4.48$ & $6.80+/-3.22$ \\
\hline & $\mathrm{CP}-4$ & $6.16+/-1.09$ & $7.17+/-3.45$ & $7.86+/-4.25$ & $7.48+/-2.93$ \\
\hline
\end{tabular}

Table 4. Expression stability ranking of the ten candidate reference genes according to geNorm, BestKeeper, NormFinder, Delta CT, and the RefFinder comprehensive analysis. These analyses were performed using the web-based RefFinder tool found at www.heartcure.com.au/reffinder/. The four genes identified by geNorm in the qbase + software as most stable are indicated in boldface. 
GeNorm

Incubation Period

Oxygen Shock and Heat Shock

Ranking Gene

1 (Most ftsZI 0.73

stable/

best)

2

3

4

5

6

8

9

10

(Least

stable)

$\begin{array}{ll}\text { gyrA } & 0.894 \\ \text { recA } & \end{array}$

IPOA 1.317

rho $\quad 1.683$

gdhA 3.463

gdhA 2.121

tpiA $\quad 4.257$

BestKeeper

All Conditions Incubation

Period

Oxygen Shock and Heat Shock

All Conditions

Gene M value Gene ST Dev Gene ST Dev Gene $\begin{aligned} & \text { ST } \\ & \text { Dev }\end{aligned}$

ftsZI 1.215

rrs $\quad 1.33$

recA 1.135

ftsZ 1.621

rho $\quad 1.49$

gyrA 1.205

gyrA 1.805

gdhA 1.86

ftsZ 1.228

recA 1.936

recA 1.768

recA 1.93

rpoA 1.561

rho 2.11

rho $\quad 1.92$

ftsZ $\quad 2.2$

rho 2.022

rrs $\quad 2.321$

$\begin{array}{llll}r p s J & 1.953 & \text { rpsJ } & 2.144\end{array}$

tpiA $\quad 2.64$

rpsJ 2.388

rpoA 2.322

$\begin{array}{llll}\text { adk } & 2.258 \quad \text { adk } & 2.348\end{array}$

gyrA 2.7

rrs

2.762

rpsJ 3.061

$\begin{array}{llll}\text { rrs } & 2.682 & \text { rrs } & 2.625\end{array}$

rpsJ 3.21

adk $\quad 3.405$

adk $\quad 3.386$

tpiA 3.392

rpoA $\quad 3.48$

gdhA 4.322

tpiA 4.305

gdhA 3.944

adk $\quad 3.62$

tpiA

5.36

gdhA $\quad 5.677$

\section{Delta CT}

Incubation Period

Oxygen Shock and Heat Shock

$\begin{array}{ll}\text { Ranking Gene Avg } & \\ \text { STDEV }\end{array}$

1 (Most ftsZ 1.56 stable/

best)

2

3

4

5

6

9

10

(Least

stable)

rpoA 3.07

gyrA $\quad 3.19$

rho $\quad 3.35$

ftsZ $\quad 3.37$

rpsJ 3.95

adk $\quad 4.09$

rrs $\quad 4.84$

gdhA $\quad 6.03$

tpiA 7.44
recA 3.26

$\operatorname{gdhA} \quad 2.79$
All Conditions

Gene Avg STDEV

gyrA 2.93

rho $\quad 3.08$

recA 3.15

ftsZ $\quad 3.2$

IPOA $\quad 3.23$

rpsJ 3.71

adk $\quad 3.73$

rrs $\quad 4.2$

tpiA $\quad 6.07$

gdhA

6.15

\section{RefFinder comprehensive ranking}

Incubation Period Oxygen Shock and Heat Shock

and Heat Shock

All Conditions

\section{Normfinder}

Incubation

Gene

Stability

value

$f t s Z \quad 0.365$

recA $\quad 0.411$

gyrA $\quad 0.53$

rho $\quad 1.086$

tpiA $\quad 1.395$

rpsJ 1.829

IPOA 1.937

rrs $\quad 1.996$

Oxygen Shock and Heat Shock

All Conditions

Gene Stability Gene value

IPOA

0.782

rho

0.303

adk 2.25

$g d h A$

2.477

\begin{tabular}{|llll} 
rho & 0.984 & gyrA & 1.158 \\
\hline gyrA & 1.523 & recA & 1.537 \\
\hline ftsZ & 1.599 & ftsZ & 1.666 \\
\hline recA & 1.811 & rpoA & 1.729 \\
\hline adk & 2.684 & adk & 2.464 \\
\hline rpsJ & 3.085 & rpsJ & 2.699 \\
\hline rrs & 3.923 & rrs & 3.209 \\
\hline gdhA & 5.215 & tpiA & 5.498 \\
\hline tpiA & 7.038 & gdhA & 5.573
\end{tabular}

$\begin{array}{llll}\text { Ranking Gene } & \begin{array}{l}\text { Geomean } \\ \text { of } \\ \text { ranking } \\ \text { values }\end{array} & \begin{array}{l}\text { Geomean } \\ \text { of } \\ \text { ranking } \\ \text { values }\end{array} & \begin{array}{l}\text { Geomean } \\ \text { of } \\ \text { ranking } \\ \text { values }\end{array}\end{array}$

Page 13/15 


\begin{tabular}{lllllll}
$\begin{array}{l}\text { (Most } \\
\text { stable) } \\
\text { best) }\end{array}$ & ftsZ & 1.50 & gyrA & 1.86 & gyrA & 1.41 \\
\hline 2 & recA & 2.00 & recA & 1.97 & ftsZ & 2.00 \\
\hline 3 & rho & 3.36 & rpoA & 2.00 & rho & 2.51 \\
\hline 4 & gyrA & 3.71 & ftsZ & 3.66 & recA & 3.22 \\
\hline 5 & rrs & 4.76 & rho & 3.76 & rpoA & 4.61 \\
\hline 6 & tpiA & 5.52 & rpsJ & 6.24 & rpsJ & 6.48 \\
\hline 7 & rpsJ & 6.45 & adk & 6.69 & adk & 6.96 \\
\hline 8 & gdhA & 7.40 & rrs & 7.74 & rrs & 7.11 \\
\hline 9 & rpoA & 7.45 & gdhA & 9.00 & tpiA & 9.00 \\
\hline $\begin{array}{l}10 \\
\text { (Least } \\
\text { stable) }\end{array}$ & adk & 9.24 & tpiA & 10.00 & gdhA & 10.00 \\
\hline
\end{tabular}

\section{Figures}

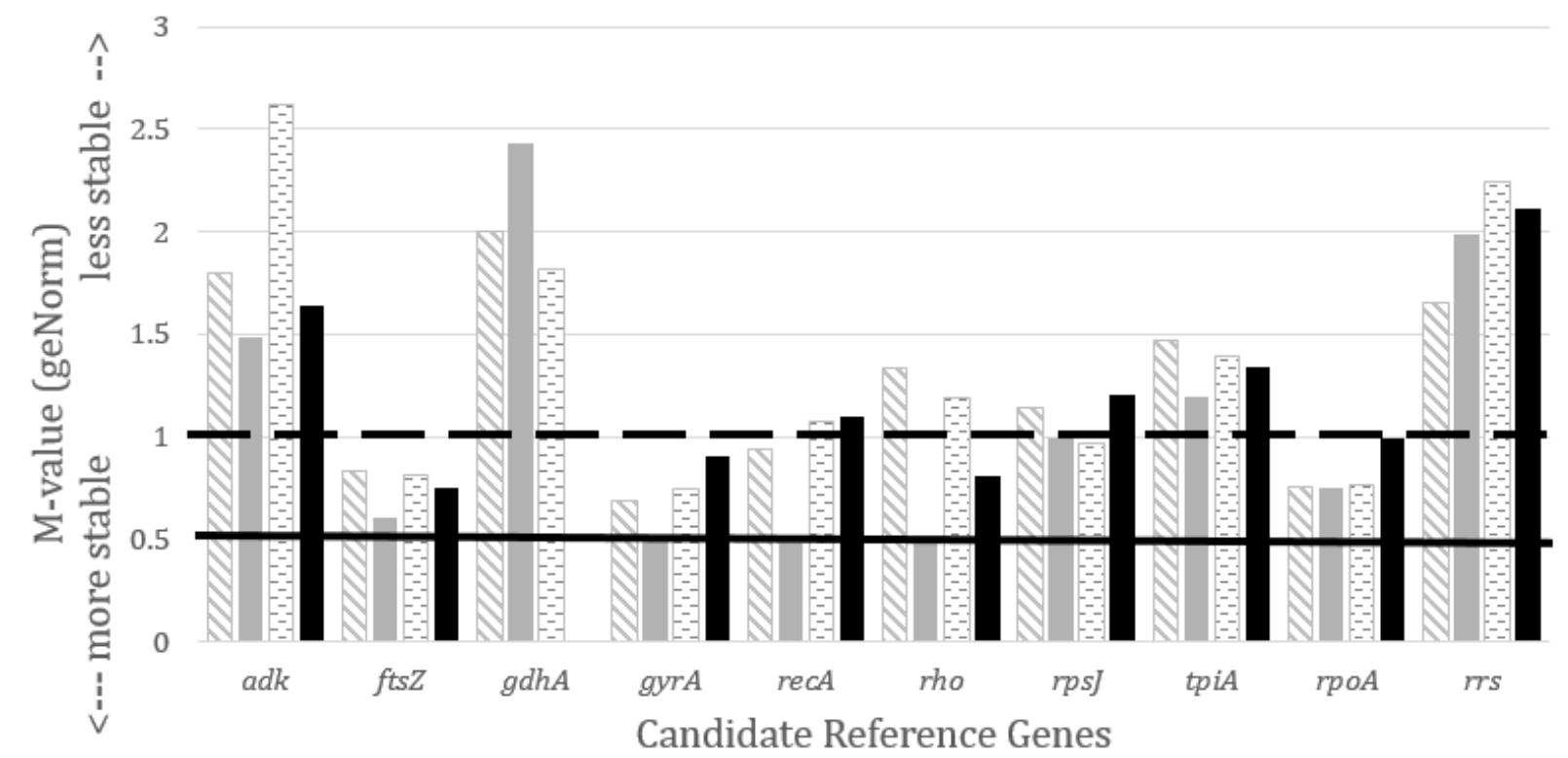

\$ Incubation period

- Oxygen Shock

G Heat Shock

- Oxygen Shock and Heat Shock Combined

\section{Figure 1}

geNorm M-values for the 10 candidate reference genes for each set of growth parameters or stress condition using qbase+ software. Medium stability $(0.5<$ average geNorm M-value $<1.0)$ is typically seen with heterogeneous samples. 


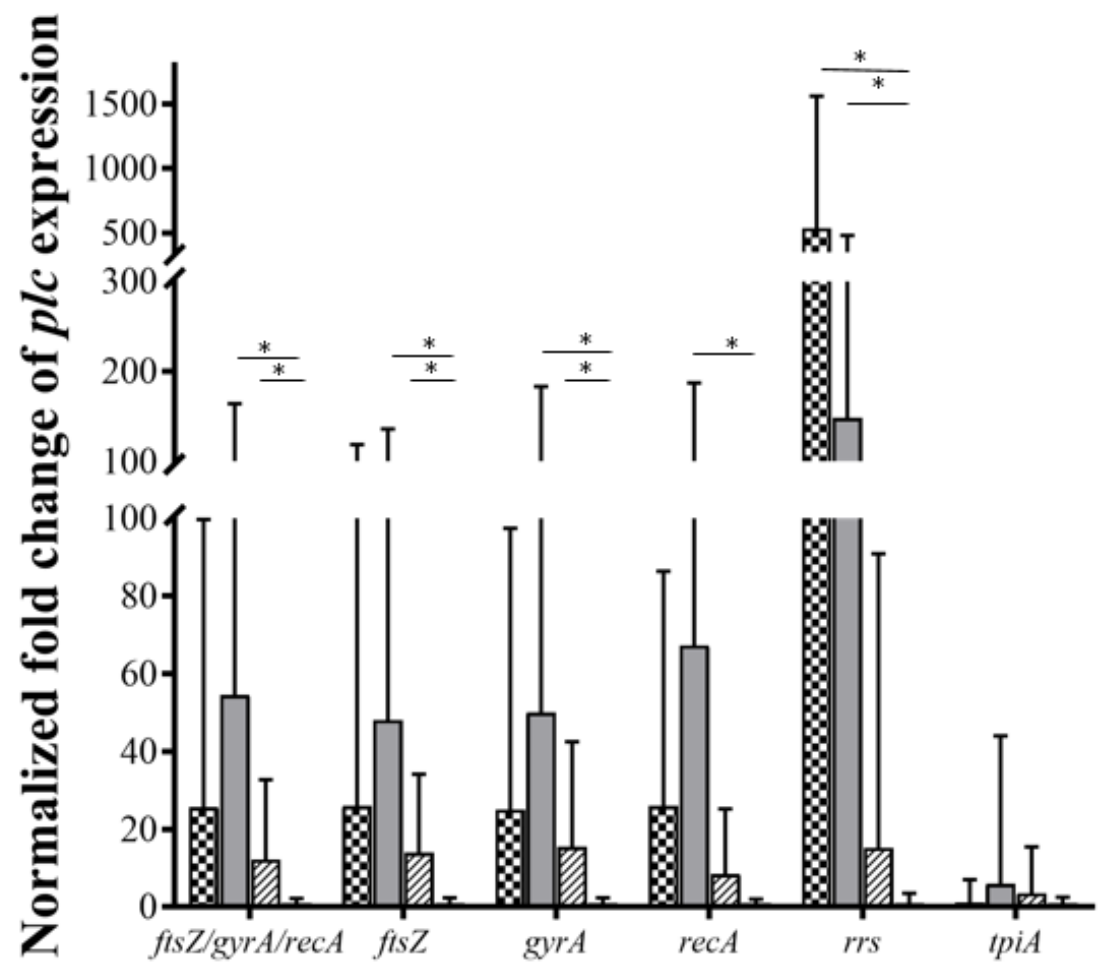

Set of genes/gene used for normalization

Q $2.5 \mathrm{hr} \square 4 \mathrm{hr} \square 6 \mathrm{hr} \square$ Overnight

Figure 2

Relative expression of alpha toxin (plc) during growth of Clostridium perfringens over a 22-hr period. The expression data were normalized using ftsZ, gyrA, recA, rrs, or tpiA as reference genes individually and with the geometric mean of ftsZ + gyrA + recA. 22-hr samples were considered the control for calculations. $P \leq 0.05$ shown by *

\section{Supplementary Files}

This is a list of supplementary files associated with this preprint. Click to download.

- SupplementaryData11172021.pdf 